\title{
Detection of epidermal growth factor receptor mutation in lung cancer by droplet digital polymerase chain reaction
}

This article was published in the following Dove Press journal:

OncoTargets and Therapy

22 June 2015

Number of times this article has been viewed

\author{
Qing $X u^{1, *}$ \\ Yazhen Zhu ${ }^{2, *}$ \\ Yali Bai' \\ Xiumin Wei' \\ Xirun Zheng ${ }^{2}$ \\ Mao Mao' \\ Guangjuan Zheng ${ }^{2}$ \\ 'Translational Bioscience and \\ Diagnostics, WuXi AppTec, Shanghai, \\ ${ }^{2}$ Department of Pathology, Guangdong \\ Provincial Hospital of TCM, \\ Guangzhou University of Chinese \\ Medicine, Guangdong Provincial \\ Academy of Chinese Medical Sciences, \\ Guangzhou, People's Republic \\ of China \\ *These authors contributed equally \\ to this work
}

Background: Two types of epidermal growth factor receptor (EGFR) mutations in exon 19 and exon 21 (ex19del and L858R) are prevalent in lung cancer patients and sensitive to targeted EGFR inhibition. A resistance mutation in exon 20 (T790M) has been found to accompany drug treatment when patients relapse. These three mutations are valuable companion diagnostic biomarkers for guiding personalized treatment. Quantitative polymerase chain reaction (qPCR)based methods have been widely used in the clinic by physicians to guide treatment decisions. The aim of this study was to evaluate the technical and clinical sensitivity and specificity of the droplet digital polymerase chain reaction (ddPCR) method in detecting the three EGFR mutations in patients with lung cancer.

Methods: Genomic DNA from H1975 and PC-9 cells, as well as 92 normal human blood specimens, was used to determine the technical sensitivity and specificity of the ddPCR assays. Genomic DNA of formalin-fixed, paraffin-embedded specimens from 78 Chinese patients with lung adenocarcinoma were assayed using both qPCR and ddPCR.

Results: The three ddPCR assays had a limit of detection of $0.02 \%$ and a wide dynamic range from 1 to 20,000 copies measurement. The L858R and ex19del assays had a $0 \%$ background level in the technical and clinical settings. The T790M assay appeared to have a $0.03 \%$ technical background. The ddPCR assays were robust for correct determination of EGFR mutation status in patients, and the dynamic range appeared to be better than qPCR methods. The ddPCR assay for T790M could detect patient samples that the qPCR method failed to detect. About $49 \%$ of this patient cohort had EGFR mutations (L858R, 15.4\%; ex19del, 29.5\%; T790M, 6.4\%). Two patients with the ex19del mutation also had a naïve T790M mutation.

Conclusion: These data suggest that the ddPCR method could be useful in the personalized treatment of patients with lung cancer.

Keywords: ddPCR, companion diagnostics, L858R, ex19del, T790M

\section{Introduction}

Lung cancer is a leading cause of cancer mortality worldwide. More than $85 \%$ of lung cancers are non-small cell lung cancer. Adenocarcinoma and squamous cell carcinoma are the two major subtypes of non-small cell lung cancer, comprising $50 \%$ and $40 \%$ of cases, respectively. ${ }^{1}$ Genomic studies such as next-generation sequencing and other high-throughput profiling platforms have identified genetic alterations common to both adenocarcinoma and squamous cell carcinoma, including in KRAS, BRAF, epidermal growth factor receptor $(E G F R)$, and ALK. ${ }^{1}$ Among these mutations or fusion genes, alterations in the EGFR have been identified in about $12 \%-13 \%$ of cases examined so far worldwide, ${ }^{1,2}$ and are much more frequent in Asian populations $(\sim 50 \%){ }^{3}$ EGFR is a member of the ERBB family of receptor tyrosine kinases involved 
in the pathogenesis of many malignant cancers. Abnormal activity of $E G F R$ results in deregulated cell proliferation and growth, which makes EGFR an important drug target in cancer cells. ${ }^{4,5}$ In fact, small-molecule tyrosine kinase inhibitors (TKIs), such as gefitinib and erlotinib, have been shown to significantly prolong the survival of lung cancer patients with EGFR-mutant tumors., ${ }^{3,6}$

Among the TKI-sensitive mutations in EGFR, a point mutation in exon 21, which substitutes an arginine for a leucine (L858R), and a small in-frame deletion in exon 19, which removes several amino acids, are the most common activating mutations that confer treatment benefits (together accounting for approximately $90 \%$ of TKI-sensitive mutations). ${ }^{6-8}$ Despite the therapeutic benefits of $E G F R$ TKIs, about $50 \%$ of patients with EGFR-mutant tumors develop acquired resistance via a second-site mutation in the threonine gatekeeper residue at position 790, ie, T790M. ${ }^{3,6,9}$ Hence, the three mutation biomarkers can be used as genetic determinants for decision-making about treatment and monitoring the treatment course.

In this study, the EGFR mutation status (L858R, ex19del, or T790M) of clinical samples from patients with lung adenocarcinoma was evaluated by two methods, ie, quantitative polymerase chain reaction (qPCR) and droplet digital polymerase chain reaction (ddPCR). On the basis of the differences observed between the two platforms, we consider that the ddPCR method can accurately determine EGFR mutation status without using standard curves or internal controls, has a wider dynamic range, and has the advantage of absolute quantification, thereby alleviating diagnostic ambiguity in the clinical setting.

\section{Materials and methods Cell lines}

EGFR wild-type A549 cells, EGFR-L858R and EGFRT790M mutation-carrying H1975 cells, and EGFR ex19del (Glu746-Ala750) mutation-carrying PC9 cells were obtained from the American Type Culture Collection (Manassas, VA, USA). Cells were cultured in their respective medium in T150 flasks until confluency and harvested with trypsin. Cell pellets were used to prepare genomic DNA using the QIAamp ${ }^{\circledR}$ DNA mini kit (Qiagen, Valencia, CA, USA) following the manufacturer's instructions.

\section{Normal genomic DNA}

Human blood was collected from healthy volunteers using the standard venipuncture procedure. Blood samples were centrifuged in BD tubes (BD Biosciences, Franklin Lakes,
NJ, USA) at $2,500 \times g$ for 10 minutes at room temperature. The plasma portion was carefully pipetted into a new $15 \mathrm{~mL}$ tube and stored at $-80^{\circ} \mathrm{C}$. The buffy coat and red cells were lysed in red blood cell lysis buffer. The white cell pellets were resuspended in phosphate-buffered saline and genomic DNA was prepared using the QIAamp DNA mini kit. The concentrations of all the DNA samples were assessed using a NanoDrop 8000 spectrophotometer (Thermo Scientific, Wilmington, DE, USA).

\section{Patient samples}

Lung tumor specimens were obtained from Guangdong Provincial Hospital of Traditional Chinese Medicine with previous fully informed consent from the patients and with approval from the hospital. The formalin-fixed, paraffinembedded (FFPE) sections were used to prepare DNA using a QIAamp DNA FFPE tissue kit (Qiagen).

\section{Real-time PCR}

Quantitative PCR was conducted with a human EGFR gene mutation detection kit (PCR fluorescence probe) kit (YQ Biomed, Shanghai, People's Republic of China) according to the manufacturer's instructions. Real-time PCR was performed using a ViiA ${ }^{\mathrm{TM}} 7$ instrument (Life Technologies, Carlsbad, CA, USA). PCR conditions were $94^{\circ} \mathrm{C}$ for 3 minutes to activate the DNA polymerase followed by 40 cycles at $94^{\circ} \mathrm{C}$ for 15 seconds and 40 cycles at $60^{\circ} \mathrm{C}$ for 1 minute. The difference in the cycle threshold $(\mathrm{Ct})$ value of the mutant versus internal control $(\Delta \mathrm{Ct})$ was used to determine the mutation status of each sample.

\section{Droplet digital PCR}

Design of the primers and probes for L858R, ex19del, and T790M was based on Oxnard et al. ${ }^{10}$ The duplex probes for ex19del detection cover the K749-S752 region and the A755D761 region, respectively, and in principle any deletion in the two areas can be detected. PCR primers and TaqMan probes (FAM-labeled or VIC-labeled) were obtained from Life Technologies. Next, $900 \mathrm{nM}$ probes and $250 \mathrm{nM}$ primers were mixed with $2 \times$ Droplet PCR Supermix (Bio-Rad Laboratories, Hercules, CA, USA), $4 \mu \mathrm{L}$ of template DNA, and $\mathrm{H}_{2} \mathrm{O}$ to become $20 \mu \mathrm{L}$ for each reaction. The reaction mixture was applied to the droplet generator with $70 \mu \mathrm{L}$ of oil to form droplets in approximately $35 \mu \mathrm{L}$ of oil-in-water mixture. The mixture was transferred to a 96-well PCR plate and heatsealed. The plate was placed in a $\mathrm{C} 1000$ Touch thermal cycler (Bio-Rad Laboratories), and amplified to the endpoint PCR. Thermal cycling conditions were $95^{\circ} \mathrm{C} \times 10$ minutes (one 
cycle), 40 cycles at $94^{\circ} \mathrm{C}$ for 30 seconds, $62^{\circ} \mathrm{C} \times 1$ minute, and $98^{\circ} \mathrm{C} \times 10$ minutes (one cycle), with a ramp rate of $1{ }^{\circ} \mathrm{C}$ per second. After PCR, the 96-well PCR plate was read on a QX-200 droplet reader (Bio-Rad Laboratories). The data were analyzed with Quantalife software. Briefly, a threshold line was drawn for channel 1 and channel 2, respectively, to separate the two clusters of negative and positive droplets. The threshold line was determined by the positive control samples, ie, genomic DNA from H1975 or PC-9. The Quantalife software calculates the copy number of both mutant and wild-type DNA based on the Poisson statistic.

\section{Results}

\section{Sensitivity and specificity of detection of EGFR mutation by ddPCR}

To determine the limit of detection of mutant DNA on the confounding background of wild-type DNA, we serially diluted 20,000 copies of H1975 or PC9 genomic DNA into 20,000 copies of normal human blood DNA (about $66 \mathrm{ng}$ ) to one copy, and measured the actual number of copies using ddPCR. All three assays showed good linearity $\left(R^{2}>0.999\right)$ and a wide dynamic range (1-20,000 copies, Figure 1). According to the statistical considerations for rare detection experimental design from the Droplet Digital ${ }^{\mathrm{TM}}$ PCR Applications Guide (Bio-Rad Laboratories), to guarantee with $95 \%$ confidence that at least one mutant molecule will be screened in the sample, statistics dictate that at least three times the number of expected wild-type target molecules must be screened (ie, number of molecules to screen $=3 \times$ background molecules). The sensitivity of these assays can be calculated to be $0.02 \%$ based on the formula (these detection limits are comparable with other digital methods, such as BEAMing [0.01\%], ${ }^{11}$ but better than qPCR-based methods such as the Scorpion amplification refractory mutation system $[0.1 \%],{ }^{12}$ wild-type quenching PCR $[0.1 \%],{ }^{13}$ and the Sequenom MassARRAY platform [0.3\%]). ${ }^{14}$

To determine the background level of the ddPCR assays, we measured mutant copy numbers in A549 cells and in 92 normal human blood genomic DNA samples from healthy volunteers. No mutant copies were detected in 20,000 copies of A549 genomic DNA. For both the L858R and ex19del assays, no mutant copies were detected in 20,000 copies of
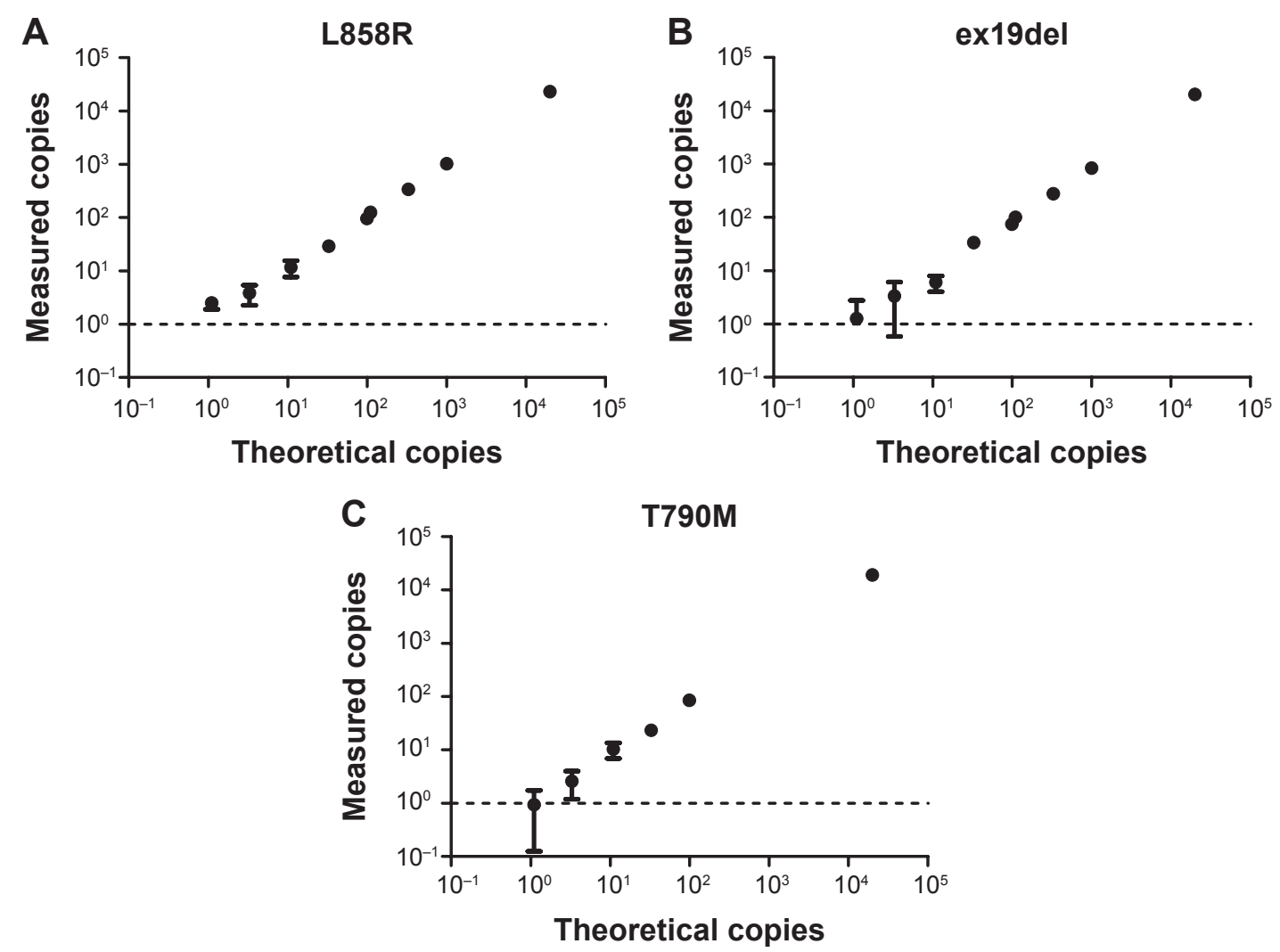

Figure I Limit dilution test to determine the sensitivity of the three EGFR mutation detection assays by the ddPCR approach.

Notes: (A) L858R assay carried out using $66 \mathrm{ng}$ of HI975 genomic DNA diluted in 20,000 copies of normal human genomic DNA. (B) The ex I9del assay carried out using $66 \mathrm{ng}$ of PC9 genomic DNA diluted in 20,000 copies of normal human genomic DNA. (C) T790M assay carried out using 66 ng of HI975 genomic DNA diluted in 20,000 copies of normal human genomic DNA. The lowest copy detected for all three assays was I.4, and thus the limit of detection was $0.015 \%$ considering the sampling errors. Abbreviations: EGFR, epidermal growth factor receptor; ddPCR, digital droplet polymerase chain reaction. 
Table I Clinical characteristics of patients

\begin{tabular}{ll}
\hline Characteristics & Patients (n) \\
\hline Sex & \\
Male (\%) & $43(55)$ \\
Female (\%) & $35(45)$ \\
Age, years & \\
$\quad$ Total median (range) & $62.5(37-91)$ \\
Male median (range) & $66(37-84)$ \\
Female median (range) & $57(37-91)$ \\
Smoking status (female) & \\
Smoker & $34(4)$ \\
Never-smoker & $40(30)$ \\
N/A* & $4(1)$ \\
Pathology & \\
Adenocarcinoma & 78 \\
Stage & \\
I-III & 27 \\
IV & 51 \\
EGFR mutation by ddPCR (L858R and exl9del) (smoker/non-smoker) \\
Male & $(5 / 6)$ \\
Female & $(3 / 21)^{* *}$ \\
\hline
\end{tabular}

Notes: *N/A, smoking status of patient is uncertain; **P=0.03 by $\chi^{2}$ test. Abbreviations: ddPCR, digital droplet polymerase chain reaction; EGFR, epidermal growth factor receptor.

normal human DNA, suggesting both assays are highly specific. In the T790M assay, we detected 1-5.2 mutant copies in 15 of 92 normal samples. Using the mean plus 3 standard deviations as the cutoff, the threshold line for false positive calls can be determined to be six copies when measuring 20,000 copies of input DNA (ie, $0.03 \%$ ).

\section{Detection of EGFR mutation status in clinical specimens}

To determine if ddPCR assays can be used to detect $E G F R$ mutations in patients with lung cancer, we screened 78 samples from resected FFPE lung tissues. The characteristics of the patients are summarized in Table 1. All the samples were categorized as adenocarcinoma. The patients were balanced in terms of sex and smoking status. The median age of the female patients was significantly lower than that of the male patients (Mann-Whitney $U$-test, one-tailed, $P=0.023$ ). About two thirds of the patients had advanced (stage IV) lung cancer.
Twelve of the samples were detected as L858R-positive, and 23 were detected as ex19del-positive (Table 2). Interestingly, two patient samples that were positive for the ex $19 \mathrm{del}$ allele were also positive for T790M mutation (data not shown), consistent with the observation of naïve mutation of T790M in patients with lung cancer. ${ }^{3}$

To determine the background level of ddPCR assays in the clinical samples, we compared samples with known L858R and ex19del mutation status based on their mutual exclusivity. For the L858R assay, no copies of mutant alleles were detected in any of the 23 ex19del-positive samples (Figure 2A); and for the ex19del assay, no copies of mutant alleles were detected in any of the 12 L858R-positive samples (Figure 2B). This result is consistent with the high specificity result obtained with 20,000 copies of normal human blood genomic DNA described above, suggesting that the two assays were highly specific in the FFPE specimens.

An advantage of ddPCR is that the allele frequency of the mutant allele can be determined quantitatively by measuring both mutant and wild-type alleles in a single PCR reaction. The allele frequency can be calculated as the mutant allele copy number divided by the sum of the mutant allele copy number and the wild-type copy number. The lowest allele frequency in the L858R sample set was 4\% (Figure 3A), whereas in the ex19del sample set it was $18 \%$ (Figure 3B). There appeared to be no correlation between mutant allele frequency and wild-type copy number for each sample in both the L858R and ex19del assays, with a Pearson correlation of $R^{2}=0.05$ and $R^{2}=0.4$, respectively (Figure 3 ). Both sample sets contained samples with allele frequencies greater than $50 \%$, suggesting that there may be amplifications of mutant alleles in these samples or that the tumor cell content was very high in the sample (eg, sample 15 in Figure 3B). In addition, the wild-type copy number may serve as a quality control for the individual testing sample.

In addition to the two positive T790M samples, we have observed eight additional samples with $>0$ copies of mutant T790M alleles. Using the threshold line of six mutant copies out of 20,000 wild-type copies defined by the normal blood

Table 2 Concordance of L858R, ex I9del, and T790M mutation detection by ddPCR with qPCR

\begin{tabular}{|c|c|c|c|c|c|c|c|c|c|}
\hline \multirow[t]{3}{*}{ ddPCR } & \multicolumn{9}{|l|}{ qPCR } \\
\hline & \multicolumn{3}{|l|}{ L858R } & \multicolumn{3}{|l|}{ exI9del } & \multicolumn{3}{|l|}{ T790M } \\
\hline & Positive & Negative & Total & Positive & Negative & Total & Positive & Negative & Total \\
\hline Positive & 12 & 0 & 12 & 23 & 0 & 23 & 2 & 3 & 5 \\
\hline Negative & 0 & 66 & 66 & 0 & 55 & 55 & 0 & 73 & 73 \\
\hline Total & 12 & 66 & 78 & 23 & 55 & 78 & 2 & 76 & 78 \\
\hline
\end{tabular}

Abbreviations: ddPCR, digital droplet polymerase chain reaction; qPCR, quantitative polymerase chain reaction. 

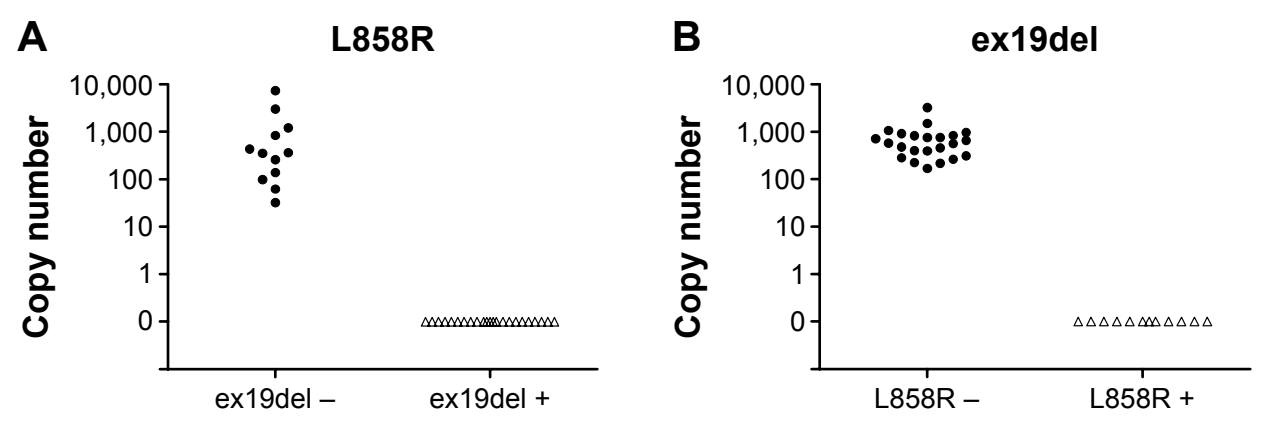

Figure 2 Background of L858R and ex I9del assays in formalin-fixed, paraffin-embedded genomic DNA.

Notes: (A) Twenty-three samples positive in ex I9del showed no copies of the L858R allele. (B) Twelve samples positive in L858R showed no copies of the ex I9del allele.

genomic DNA (or $0.03 \%$ ), we could rule out three samples as false positives (data not shown). When examining the two-dimensional plot of the data, we found that two samples only showed droplets in the second quadrant (Figure 4A). Based on the sample partitioning principle of ddPCR, it is not possible that these droplets are from a true mutant allele. Instead, they are most likely from cross-hybridization of the mutant probe with the wild-type DNA in the sample. We then repeated the remaining three samples and found that they consistently showed a mutant allele frequency $>0.03 \%$ (Figure 4B). When examining the two-dimensional plot, they showed positive droplets mainly in the first quadrant (Figure 4C). These three samples, although with a low mutant allele frequency, are very likely to be true positives.

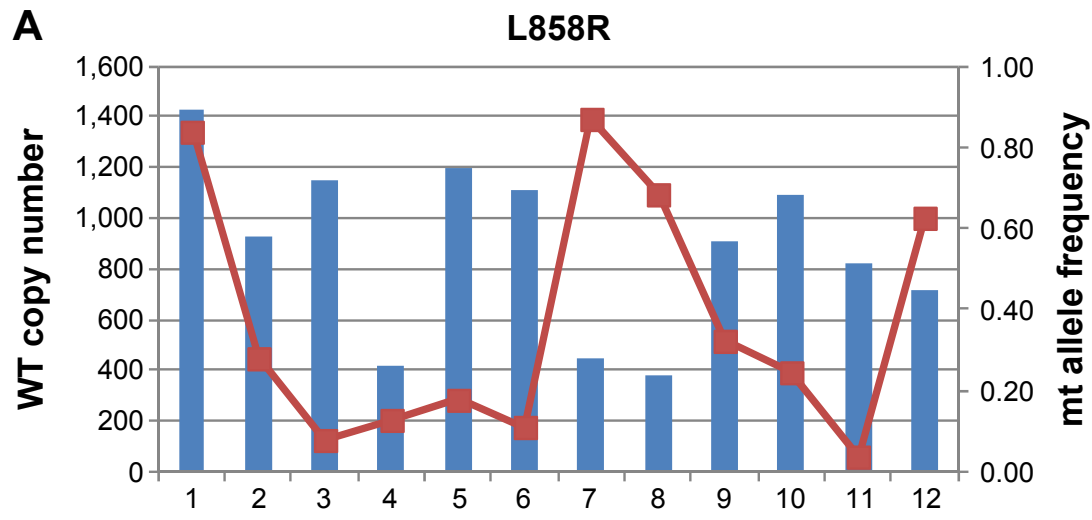

B

ex19del

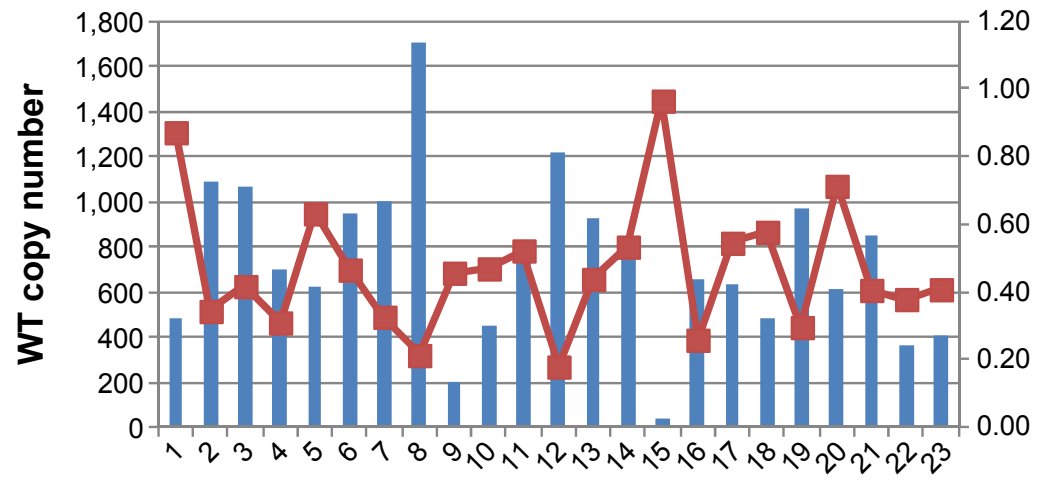

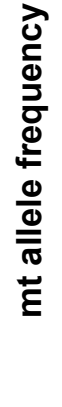

WT copy number $\quad-$ mt allele frequency

Figure 3 WT copy number and mutant allele frequency distribution of L858R and ex I9del assays.

Notes: The WT copy number was obtained directly from each assay reaction readout. The frequency of the mutant allele was calculated by the copy number of the mutant allele/(copy number of mutant allele + copy number of WT allele). (A) L858R assay and (B) ex I 9del assay.

Abbreviations: WT, wild-type; $\mathrm{mt}$, mutant. 

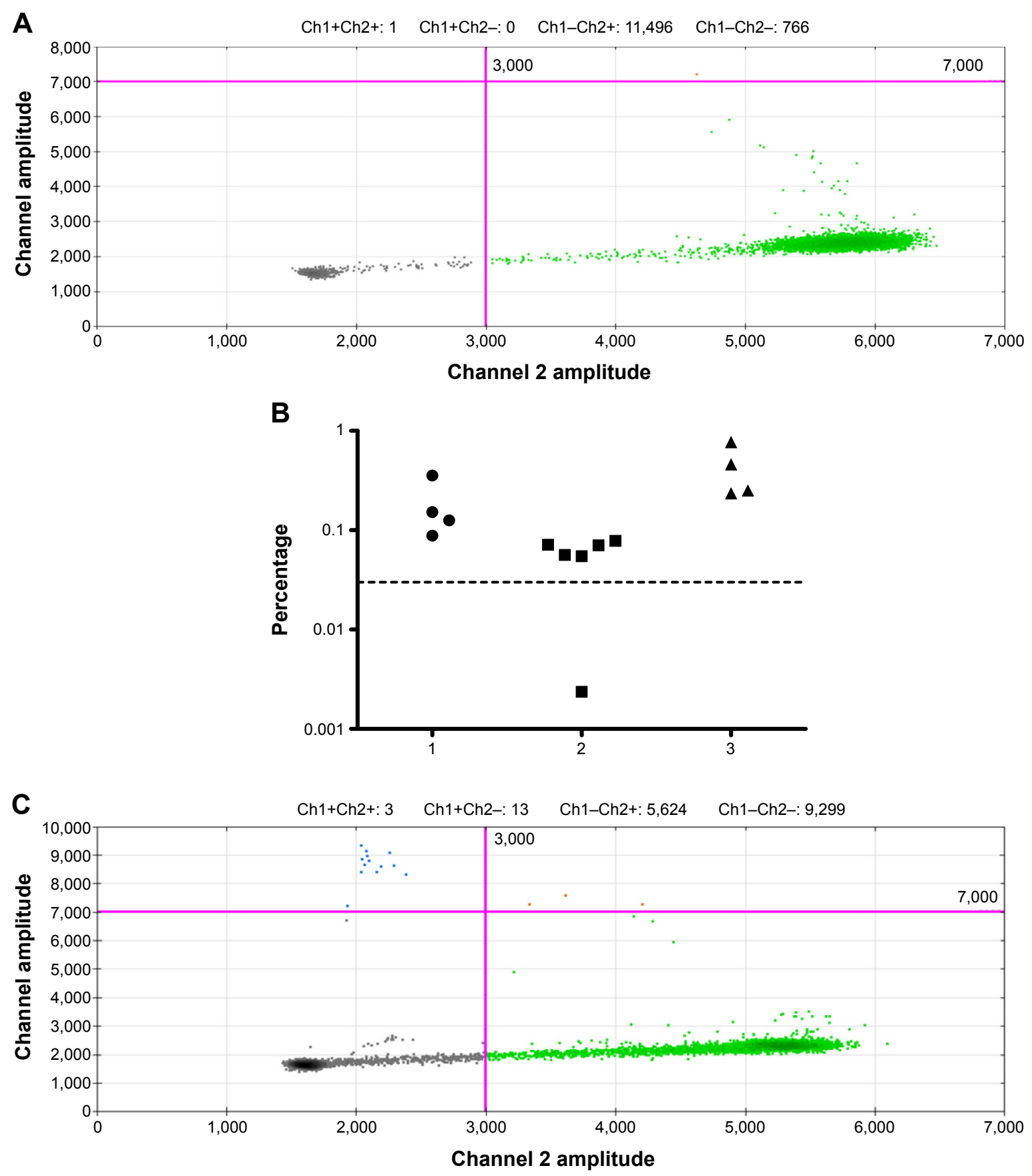

Figure 4 Determination of true positives for T790M in formalin-fixed, paraffin-embedded genomic DNA.

Notes: (A) Example of two-dimensional plot of a false positive sample in the T790M assay using formalin-fixed, paraffin-embedded genomic DNA. The positive signal comes from the second quadrant only. (B) Three reproducible samples showing allele frequency above the $0.03 \%$ threshold (dotted line), which were determined as negative in the quantitative polymerase chain reaction assay. (C) Example of a two-dimensional plot of a true positive sample in the T790M assay using formalin-fixed, paraffin-embedded genomic DNA. The main positive signal comes from the first quadrant.

To understand how accurately ddPCR methods can capture mutation status in patients with lung cancer, we compared the results with qPCR results previously obtained in the pathology laboratory when patients were diagnosed with lung cancer. The qPCR data were obtained with commercial kits based on the Scorpion amplification refractory mutation system method. ${ }^{12,15}$ The L858R and ex19del assays showed
$100 \%$ concordance with qPCR results in detecting positive and negative samples; however, three samples determined as positive in the ddPCR assay results for T790M were negative in the qPCR assay results (Table 2). The concordance rate of the T790M assay was $96 \%$. These data suggest that the ddPCR assay for T790M is more sensitive than the qPCR assay in clinical samples. 


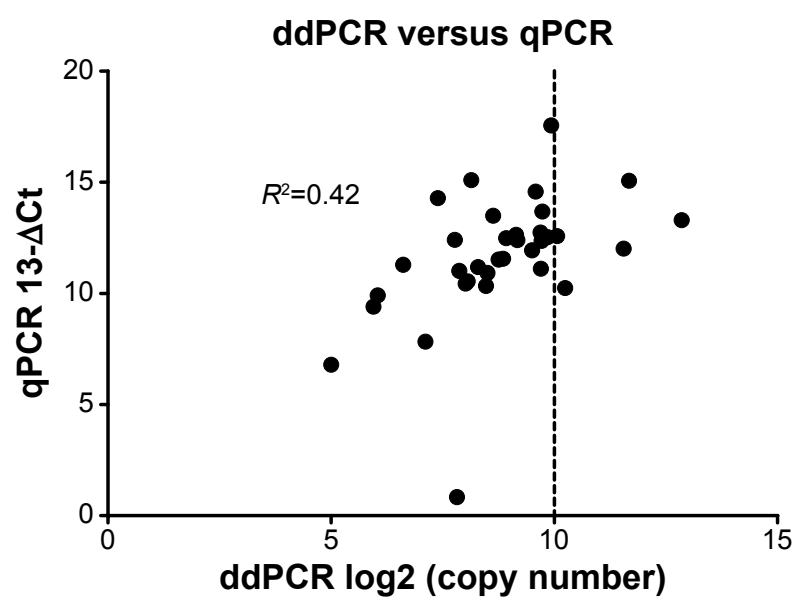

Figure 5 Correlation between $\triangle \mathrm{Ct}$ and copy numbers measured by ddPCR. Thirtyfive patients positive for EGFR mutations had both $\triangle \mathrm{Ct}$ from the $\mathrm{qPCR}$ assay and the absolute copy number from ddPCR assay. A good correlation is observed when the copy number is relatively small.

Abbreviations: $d d P C R$, digital droplet polymerase chain reaction; qPCR, quantitative polymerase chain reaction; EGFR, epidermal growth factor receptor.

\section{Quantitative comparison of ddPCR with qPCR}

Digital PCR has the advantage of measuring absolute copy numbers without using the standard curve. ${ }^{16}$ In the qPCRbased method, a standard curve is necessary to measure the copy numbers of test samples, but can introduce extra efforts and errors. In addition, commercial assays sometimes only use the relative quantitation method, ie, the $\Delta \mathrm{Ct}$ with the reference gene to determine the sample mutation status as either positive or negative. To find out whether the absolute copy numbers determined by ddPCR can recapitulate the mutation level measured by the qPCR method, we compared the copy numbers in log scale with the $\Delta \mathrm{Ct}$ measured for each patient sample. The two values showed relatively good correlation when the mutant copy numbers were relatively low (Figure 5). The $R^{2}$ value was 0.42 if the high copy number points were not considered. However, the $\Delta \mathrm{Ct}$ method showed a plateau with the increase in mutant copy number, suggesting that the ddPCR method has a better dynamic range than the qPCR method when a standard curve is not applied (Figure 5).

\section{EGFR mutation spectrum in the lung cancer patient cohort}

To test if the ddPCR methods could capture the EGFR mutation status in lung cancer patients, we grouped the samples into EGFR mutant and EGFR wild-type classes. About $49 \%$ of samples from this patient cohort contained one or two of the three mutations (Figure 6), which is consistent with the results of previous large-scale studies of the EGFR mutation frequency in Asian patients with lung cancer $(36 \%-41 \%) .{ }^{17,18}$ To further understand the mutation frequency of the individual mutation, we analyzed the $E G F R$ mutant subset of patients and found that L858R and ex19del mutations were mutually exclusive and their mutation frequencies were $32 \%$ and $60 \%$, respectively (Figure 6). Although this result is different from the findings of the two earlier studies in the Asian population, ${ }^{17,18}$ it is consistent with observations elsewhere that the ex19del mutation is more frequent than the L858R mutation. ${ }^{13,19}$

Two patients who harbored the ex19del mutation also had the T790M mutation, and three patients harbored the T790M mutation alone. This low frequency $(5 / 78,6.4 \%)$ is consistent with the rare event of germline mutation of this predisposing allele, ${ }^{6,20}$ or sometimes linked to primary resistance, occurring together with a sensitizing mutation prior to drug exposure. ${ }^{3}$ It is worth noting that this primary mutation may have a different incidence in our particular patient cohort; for instance, more than $50 \%$ of pretreatment T790M mutations have been observed in certain lung cancer patient cohorts. ${ }^{21,22}$

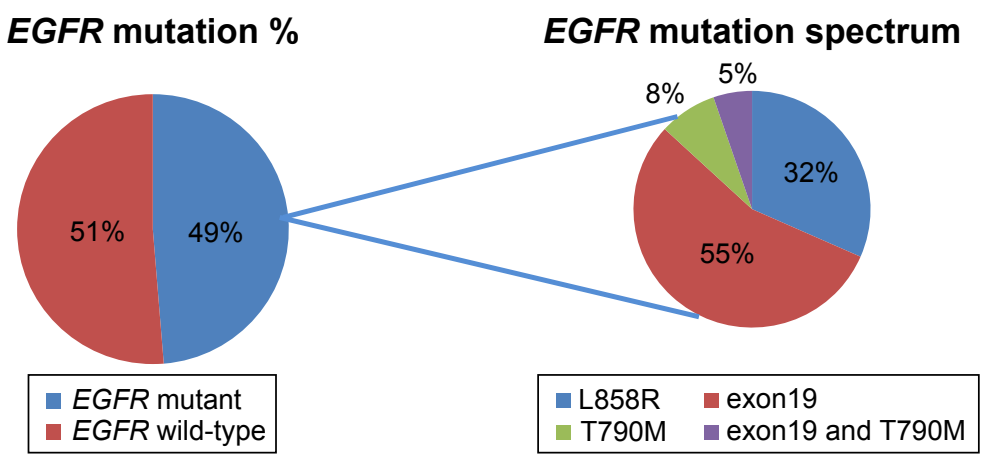

Figure 6 EGFR mutation spectrum in this 78-patient cohort. Fifty-one percent of patients (EGFR wild-type, 43/78) showed no mutation in any of the L858R, exI9del, or T790M alleles, while $49 \%$ of patients (EGFR mutant, 35/78) showed EGFR mutation in the L858R, exI 9del, or T790M alleles; among these, 32\% were L858R, 60\% were exI 9del, and $13 \%$ were T790M mutations. Two patients harbored both ex I9del and T790M mutations (5\%).

Abbreviation: EGFR, epidermal growth factor receptor. 
Thirty of the 40 never-smokers in this cohort were women. Among the 35 patients positive for the L858R or ex19del mutation, 21 were female never-smokers (Table 1). In this group of patients, the EGFR mutation was significantly more common in female never-smokers ( $\chi^{2}$ test, $P=0.03$ ), which is consistent with previous findings.

\section{Discussion}

Since its discovery about a decade ago, ${ }^{7,8}$ the relationship between the effectiveness of gefitinib in lung cancer patients and $E G F R$ mutation has become a paradigm for the success of targeted therapy in solid tumors. Many methods have since been developed, aiming to select patients based on the somatic mutation status of the EGFR gene. ${ }^{15,23-27}$ Direct sequencing and real-time PCR-based methods are most commonly used in the pathology departments of hospitals or clinical testing laboratories. However, despite their routine use, the direct sequencing method has limited utility, including low sensitivity and dependence on tumor cell content to detect mutations, and real-time PCR-based methods are semiquantitative in that a standard curve and reference control genes are required to determine the mutant copies. The digital PCR concept was proposed a while ago as a way of circumventing these limitations, ${ }^{28}$ and many studies have shown that digital PCR technology is superior for detection of rare mutation sequences on the background of confounding normal sequences in an absolute quantification manner. ${ }^{10,11,29}$

Among the major platforms currently available for digital PCR, ${ }^{30}$ we chose the Bio-Rad ddPCR platform to investigate the clinical utility in the detection of EGFR mutation. The L858R and ex19del assays can detect as low as one mutant copy among 20,000 copies of normal DNA, and no mutant signal showed up when 92 normal DNA samples are examined, suggesting that this is a highly sensitive and specific assay. In addition, the ex19del assay uses a principle of the "indirect" nature of mutant calling, ${ }^{10}$ and this nature brings a possibility of detecting mutation or deletion in the place where the wild-type probe locates (ie, EGFR aa 756N-761D). In fact, we found one particular FFPE sample having such a mutation, which was confirmed by Sanger sequencing (data not shown).

The T790M assay shows good sensitivity, but the background noise is relatively high. By examining the two-dimensional plot of normal DNA and pure $\mathrm{H}_{2} \mathrm{O}$ data, we think the background signal may come from non-specific amplification of normal DNA by the mutant probe or from primer/probe interference. By setting a threshold line of six copies per 20,000 normal copies (or $0.03 \%$ ), and constructing a two-dimensional plot review, we could eliminate the false positives in the clinical samples tested. In fact, we observed such a background "mutant" signal in eight of 78 samples, with five samples being able to be clearly ruled out as false positives by the two criteria above (three samples $<0.03 \%$, two samples showing droplets in quadrant 2 ). For the remaining three samples, we repeated the tests multiple times and found they were true positive samples that were not detected with the qPCR method, suggesting that the ddPCR assay is more sensitive in low abundance mutation detection. The technical background threshold of $0.03 \%$ based on the fresh normal genomic DNA samples may be an underestimate of the FFPE clinical samples in terms of the T790M assay; the reproducible clinical sample with a low allele frequency may be a better estimate of the background. For example, sample 2 in Figure 4 consistently showed an allele frequency above $0.05 \%$, which may be a more clinically meaningful cut-off for true positives in patients.

The concordance of L858R and ex19del assays between qPCR and ddPCR is $100 \%$, and is $96 \%$ for the T790M assay, suggesting that ddPCR can be a reliable alternative to the qPCR method. In addition, when comparing the $\Delta \mathrm{Ct}$ with the absolute copy number of the same sample, we found that ddPCR has a better dynamic range than qPCR. This is important because the $\Delta \mathrm{Ct}$ method of qPCR often gives ambiguous results when the mutant DNA is in low abundance in the test sample. Moreover, absolute copy number determination by ddPCR is accurate and convenient in terms of mutation calling in the clinical settings. The essence of a duplex assay can easily determine the abundance of mutant genes in a specimen, which can not only use smaller amounts of the clinical samples, but can also be used as a quantitative surrogate biomarker for the benefit of targeted therapeutics. ${ }^{31}$

In summary, we demonstrated that the ddPCR method was easier to interpret without standard curves and internal controls than the qPCR method when used to detect three EGFR mutations in lung cancer patients. Using a cohort of 78 Chinese patients with lung cancer, we recapitulated previous findings with regard to frequency of $E G F R$ L858R, ex19del, and T790M mutations ${ }^{3,13,17-19}$ using both qPCR and ddPCR methods. The ddPCR method can identify low abundance mutation events than qPCR. These results suggest that ddPCR can be used reliably in the clinical setting to guide patient stratification.

\section{Acknowledgments}

This research was supported by the National Natural Science Foundation of China (81202960). We thank Dr Yuhong Shen of WuXi AppTec and her team for providing the cell lines used in this study. We thank Ms Louise Mao for proofreading the manuscript. We also appreciate the volunteers at $\mathrm{WuXi}$ 
AppTec for providing the normal blood DNA samples used in this research.

\section{Disclosure}

The authors report no conflicts of interest in this work.

\section{References}

1. Chen Z, Fillmore CM, Hammerman PS, Kim CF, Wong KK. Non-smallcell lung cancers: a heterogeneous set of diseases. Nat Rev Cancer. 2014;14(8):535-546.

2. Pao W, Hutchinson KE. Chipping away at the lung cancer genome. Nat Med. 2012;18:349-351.

3. Sharma SV, Bell DW, Settleman J, Haber DA. Epidermal growth factor receptor mutations in lung cancer. Nat Rev Cancer. 2007;7(3):169-181.

4. Herbst RS, Fukuoka M, Baselga J. Gefitinib - a novel targeted approach to treating cancer. Nat Rev Cancer. 2004;4(12):956-965.

5. Tebbutt N, Pedersen MW, Johns TG. Targeting the ERBB family in cancer: couples therapy. Nat Rev Cancer. 2013;13(9):663-673.

6. Pao W, Chmielecki J. Rational, biologically based treatment of EGFR-mutant non-small-cell lung cancer. Nat Rev Cancer. 2010;10(11):760-774.

7. Lynch TJ, Bell DW, Sordella R, et al. Activating mutations in the epidermal growth factor receptor underlying responsiveness of non-small-cell lung cancer to gefitinib. N Engl J Med. 2004;350(21):2129-2139.

8. Paez JG, Jänne PA, Lee JC, et al. EGFR mutations in lung cancer: correlation with clinical response to gefitinib therapy. Science. 2004;304(5676):1497-1500.

9. Yu HA, Riely GJ, Lovly CM. Therapeutic strategies utilized in the setting of acquired resistance to EGFR tyrosine kinase inhibitors. Clin Cancer Res. 2014;20(23):5898-5907.

10. Oxnard GR, Paweletz CP, Kuang Y, et al. Noninvasive detection of response and resistance in EGFR-mutant lung cancer using quantitative next-generation genotyping of cell-free plasma DNA. Clin Cancer Res. 2014;20(6):1698-1705.

11. Taniguchi K, Uchida J, Nishino K, et al. Quantitative detection of EGFR mutations in circulating tumor DNA derived from lung adenocarcinomas. Clin Cancer Res. 2011;17(24):7808-7815.

12. Kimura H, Fujiwara Y, Sone T, et al. High sensitivity detection of epidermal growth factor receptor mutations in the pleural effusion of non-small cell lung cancer patients. Cancer Sci. 2006;97(7):642-648.

13. Nakamura T, Sueoka-Aragane N, Iwanaga K, et al. Application of a highly sensitive detection system for epidermal growth factor receptor mutations in plasma DNA. J Thorac Oncol. 2012;7(9):1369-1381.

14. Sakai K, Horiike A, Irwin DL, et al. Detection of epidermal growth factor receptor T790M mutation in plasma DNA from patients refractory to epidermal growth factor receptor tyrosine kinase inhibitor. Cancer Sci. 2013;104(9):1198-1204.

15. Xu F, Wu J, Xue C, et al. Comparison of different methods for detecting epidermal growth factor receptor mutations in peripheral blood and tumor tissue of non-small cell lung cancer as a predictor of response to gefitinib. Onco Targets Ther. 2012;5:439-447.

16. Hindson CM, Chevillet JR, Briggs HA, et al. Absolute quantification by droplet digital PCR versus analog real-time PCR. Nat Methods. 2013;10(10):1003-1005.

OncoTargets and Therapy

\section{Publish your work in this journal}

OncoTargets and Therapy is an international, peer-reviewed, open access journal focusing on the pathological basis of all cancers, potential targets for therapy and treatment protocols employed to improve the management of cancer patients. The journal also focuses on the impact of management programs and new therapeutic agents and protocols on
17. Kosaka T, Yatabe Y, Endoh H, Kuwano H, Takahashi T, Mitsudomi T. Mutations of the epidermal growth factor receptor gene in lung cancer: biological and clinical implications. Cancer Res. 2004;64(24):8919-8923.

18. Wu JY, Yu CJ, Chang YC, Yang CH, Shih JY, Yang PC. Effectiveness of tyrosine kinase inhibitors on "uncommon" epidermal growth factor receptor mutations of unknown clinical significance in non-small cell lung cancer. Clin Cancer Res. 2011;17(11):3812-3821.

19. Rosell R, Moran T, Queralt C, et al. Screening for epidermal growth factor receptor mutations in lung cancer. $N$ Engl J Med. 2009;361(10): 958-967.

20. Bell DW, Gore I, Okimoto RA, et al. Inherited susceptibility to lung cancer may be associated with the T790M drug resistance mutation in EGFR. Nat Genet. 2005;37(12):1315-1316.

21. Costa C, Molina MA, Drozdowskyj A, et al. The impact of EGFR T790M mutations and BIM mRNA expression on outcome in patients with EGFR-mutant NSCLC treated with erlotinib or chemotherapy in the randomized phase III EURTAC trial. Clin Cancer Res. 2014;20(7):2001-2010.

22. Fujita Y, Suda K, Kimura H, et al. Highly sensitive detection of EGFR T790M mutation using colony hybridization predicts favorable prognosis of patients with lung cancer harboring activating EGFR mutation. J Thorac Oncol. 2012;7(11):1640-1644.

23. Ellison G, Zhu G, Moulis A, Dearden S, Speake G, McCormack R. EGFR mutation testing in lung cancer: a review of available methods and their use for analysis of tumour tissue and cytology samples. J Clin Pathol. 2013;66(2):79-89.

24. Ho HL, Chang FP, Ma HH, et al. Molecular diagnostic algorithm for epidermal growth factor receptor mutation detection in Asian lung adenocarcinomas: comprehensive analyses of 445 Taiwanese patients with immunohistochemistry, PCR-direct sequencing and Scorpion/ ARMS methods. Respirology. 2013;18(8):1261-1270.

25. Kimura H, Kasahara K, Kawaishi M, et al. Detection of epidermal growth factor receptor mutations in serum as a predictor of the response to gefitinib in patients with non-small-cell lung cancer. Clin Cancer Res. 2006;12(13):3915-3921.

26. Kuang Y, Rogers A, Yeap BY, et al. Noninvasive detection of EGFR T790M in gefitinib or erlotinib resistant non-small cell lung cancer. Clin Cancer Res. 2009;15(8):2630-2636.

27. Shi Yeen TN, Pathmanathan R, Shiran MS, Ahmad Zaid FA, Cheah YK. Detection of epidermal growth factor receptor mutations in formalin fixed paraffin embedded biopsies in Malaysian non-small cell lung cancer patients. J Biomed Sci. 2013;20:22.

28. Vogelstein B, Kinzler KW. Digital PCR. Proc Natl Acad Sci U S A. 1999;96(16):9236-9241.

29. Yung TK, Chan KC, Mok TS, Tong J, To KF, Lo YM. Single-molecule detection of epidermal growth factor receptor mutations in plasma by microfluidics digital PCR in non-small cell lung cancer patients. Clin Cancer Res. 2009;15(6):2076-2084.

30. Day E, Dear PH, McCaughan F. Digital PCR strategies in the development and analysis of molecular biomarkers for personalized medicine. Methods. 2013;59(1):101-107.

31. Zhou Q, Zhang XC, Chen ZH, et al. Relative abundance of EGFR mutations predicts benefit from gefitinib treatment for advanced nonsmall-cell lung cancer. J Clin Oncol. 2011;29(24):3316-3321.

patient perspectives such as quality of life, adherence and satisfaction. The manuscript management system is completely online and includes a very quick and fair peer-review system, which is all easy to use. Visit http://www.dovepress.com/testimonials.php to read real quotes from published authors.

\section{Dovepress}

